\title{
Adaptive Edge-Preserving Image Denoising using Arbitrarily Shaped Local Windows in Wavelet Domain
}

\author{
Paras Jain \\ Jaypee University of Engineering \& Technology \\ Raghogarh, Guna 473226, MP, India
}

\begin{abstract}
Image denoising is a well explored topic in the field of image processing. A denoising algorithm is designed to suppress the noise while preserving as many image structures and details as possible. This paper presents a novel technique for edgepreserving image denoising using wavelet transforms. The multi-level decomposition of the noisy image is carried out to transform the data into the wavelet domain. An adaptive thresholding scheme which employs arbitrary shaped local windows and is based on edge strength is used to effectively reduce noise while preserving significant features of the original image. The experimental results, compared to other approaches, prove that the proposed method is suitable for various image types corrupted by Gaussian noise.
\end{abstract}

\section{Keywords}

Wavelet transform; arbitrary shaped window; region-based approach; noise reduction; edge-preservation.

\section{INTRODUCTION}

Image denoising is the process of obtaining the original image by reducing unwanted noise from a corrupted image. Various techniques [1-3] for noise reduction have been introduced in last few decades, many of them are based on linear spatial domain filters. Most linear filtering techniques proposed so far need some prior knowledge about the noise and the image characteristics. Usually, such details are not available and may be hard to estimate from the input noisy image. Linear filters are easy to implement and usually smooth the data to eliminate the noise; however, this process can produce blurring in images [3].

To handle the problems of linear filters, many non-linear edge-preserving methods have been developed and research is still continued in this direction [4]. These non-linear edgepreserving filtering methods can remove the noise more effectively while preserving the important image features such as edges. Wavelet-based non-linear methods have shown excellent potential in providing efficient edge-preserving image denoising since they provide a suitable basis for separating noisy signal from the image signal. The common approach for noise reduction in wavelet domain in to determine the multiscale wavelet decomposition of the noisy image and to modify the wavelet coefficients, thus obtained. Coefficients that are supposed to be noisy are modified by means of thresholding. Reconstruction from these modified coefficients then gives the desired denoised image. Numerous denoising methods follow such procedure of wavelet thresholding [5-14].

A major challenge in the wavelet shrinkage process is to estimate an appropriate threshold. Some well-known threshold estimation criteria are VisuShrink (non-adaptive) [6], SureShrink [7] (adaptive), BayesShrink [5, 17] (adaptive) and Cross Validation $[18,19]$ (adaptive). Once an appropriate threshold is estimated, the wavelet coefficients can be thresholded according to a shrinkage rule. A shrinkage

\author{
Vipin Tyagi \\ Jaypee University of Engineering \& Technology \\ Raghogarh, Guna 473226, MP, India
}

(thresholding) rule at stage 3 defines the applicability of a threshold. The ultimate goal of a shrinkage rule is to preserve large coefficients which represent important signal features, while small coefficients can be thresholded without affecting the significant image features. Often used shrinkage rules are 'keep-or-kill' hard thresholding and 'shrink-or-kill' soft thresholding [16].

Most of the wavelet-based denoising methods often require the knowledge about the variances of signal and/or noise. Since these variances are usually unknown, assumptions are frequently made. A common solution to the problem is to estimate the variance from the data. Such variance estimation is popular for various denoising methods and achieves efficient denoising results with a low complexity. Numerous denoising approaches [10-13, 20] have taken benefit of joint statistical relationships among the wavelet coefficients by estimating the variance of a coefficient adaptively from a local neighborhood window consisting of coefficients within a subband. The size of the locally adaptive window also plays an important role in estimating the signal variance.

In this paper, we present a new technique for noise reduction using wavelet transforms. A new locally adaptive thresholding scheme which involves estimation of thresholding parameters in arbitrarily shaped local neighborhood windows and is based on edge strength, is used to effectively suppress Gaussian noise while preserving relevant features of the original image. Experimental results show that the proposed method, when compared to well-known state-of-the-art denoising methods, is more suitable for various classes of images corrupted by Gaussian noise.

The motivation behind using the concept of arbitrarily shaped (varying sized) local windows has come from the denoising approach suggested by Eom and Kim [11]. In [11], authors have been established that a locally adaptive window of nearly arbitrarily shape (i.e. varying size) can be more efficient in removing white Gaussian noise in comparison of fixed size local windows. The reason being with nearly arbitrarily shaped window, one can obtain more accurate local statistics of images. However, they had pointed that denoising results obtained with their approach were not so good in higher noise environment. Through the proposed approach, we have got better denoising results with effective edgepreservation.

The remainder of this paper is organized as follows. Section 2 gives a brief review of related techniques; Section 3 contains the proposed method in detail; Section 4 gives experimental results, including a comparison with other denoising methods; Finally Section 5 summarizes the conclusions.

\section{RELATED WORK}

A number of denoising approaches in wavelet domain have used the mechanism of locally adaptive windows. In this mechanism one can estimate the variance of each wavelet 
coefficient from the coefficients in its local neighborhood. A noisy image in wavelet domain is modeled as

$$
\mathbf{y}=\mathbf{w}+\mathbf{n}
$$

where $\mathbf{y}$ is the observed image, $\mathbf{w}$ is the unknown original image and $\mathbf{n}$ is assumed to be an i.i.d. white Gaussian noise with zero mean and finite variance $\sigma_{\text {noise }}^{2}$. The goal is to recover $\mathbf{w}$ from the noisy observation $\mathbf{y}$. Several denoising approaches $[8,10-13]$ use the signal variance $\sigma_{\text {signal }, k}^{2}$ of a wavelet coefficient $w_{k}$, that is assumed to be an independent Gaussian variable. It is assumed that $\sigma_{\text {signal }, k}^{2}$ is deterministic and known. But if in fact $\sigma_{\text {signal }, k}^{2}$ is not known, $\hat{\sigma}_{\text {signal }, k}^{2}$, which is an estimate of $\sigma_{\text {signal }, k}^{2}$, should be used. Obviously, $\hat{\sigma}_{\text {signal }, k}^{2}$ has to be estimated from the given noisy data. The performance of a denoising method is dependent on the quality of the estimator, $\hat{\sigma}_{\text {signal }, k}^{2}$.

Mihcak et al. [10] proposed a powerful denoising scheme with low-complexity by exploiting the dependency of the local wavelet coefficients within each scale. In [10], authors obtained reasonably good results by performing a locally adaptive window-based maximum likelihood (LAWML) estimate. Eom and Kim [11] demonstrated improvisation over LAWML approach, as they obtained more better results by performing a nearly arbitrarily shaped window-based maximum likelihood (NASWML) estimate.

Sendur and Selesnick [8] proposed a locally adaptive thresholding method in which they had also the same estimator for signal variance of a wavelet coefficient as was used in [10]. But unlike the method proposed in [10], Sendur and Selesnick used a bivariate shrinkage function which exploits the interscale dependency between wavelet coefficients.

Jain and Tyagi [12] have recently proposed a denoising technique in tetrolet (Haar-type wavelet) domain which is deeply inspired by locally adaptive thresholding approach presented by Sendur and Selesnick [8]. They proposed a locally adaptive thresholding method which exploits interscale statistical dependency and based on computation of noise level.

Jain and Tyagi [13] have also proposed very recently a new technique for noise reduction in wavelet domain. They presented a new locally adaptive patch-based (LAPB) thresholding scheme that relies on the aggregation of multiple thresholded estimates of a wavelet coefficient and involves estimation of thresholding parameters for a wavelet coefficient (such as signal variance) in a local neighborhood.

Although the computation load is low for each coefficient in all the above discussed denoising approaches but all of them focus only on a fixed window size for the local neighborhood. Apart from this, it is a common thinking that the larger the size of the region in which to estimate the signal variance is, the more reliable this estimation is. However, the locally i.i.d assumption becomes inaccurate as the size of the neighborhood grows [20]. This suggests the existence of a proper neighborhood region for the variance estimation of each wavelet coefficient. A number of methods [11, 15, 2023] based on varying local varying windows have been developed.

\section{PROPOSED METHOD}

In previous section we have discussed the importance of using "proper neighborhood region" over fixed size neighborhood region for the variance estimation of each wavelet coefficient. By proper neighborhood region, we mean the neighborhood region in which one can obtain local statistics of images more accurately. This fact motivates us to use variable-sized neighborhood regions of nearly arbitrarily shapes (instead of fix-sized square shape). To employ the mechanism of nearly arbitrarily shaped windows, our approach used the region merging method suggested by Eom and Kim [11]. In our approach, an adaptive threshold is computed in neighborhooddependent manner to effectively characterize local features of the image. A new locally adaptive thresholding scheme is proposed to threshold the small wavelet coefficients considered to be noise while preserving edges. This neighborhood-dependent thresholding is obtained based on the calculation of local noise variance and edge strength.

Though, the proposed thresholding scheme is inspired by the arbitrarily shaped window-based locally adaptive denoising method proposed by Eom and Kim [11] but there are some differences that make it superior. First, as our scheme computes the noise variance which is used in the computation of thresholds, locally at each resolution scale, it achieves better denoising results specially in higher noise environment. Second, as our scheme considers edge strength of a pixel for the estimation of local threshold, it achieves better edgepreservation.

Apart from improvisation over denoising approach in [11], our method also outperforms locally adaptive patch-based (LAPB) thresholding scheme proposed by Jain and Tyagi [13] due to some genuine facts. First, unlike the fix-sized local neighborhood mechanism used in LAPB method, proposed approach uses variable-sized neighborhood region for the estimation of signal variance of each wavelet coefficient. Second, the scheme preserves edges more efficiently as it considers the edge strength of a pixel during its thresholding. The main stages of the proposed denoising method are illustrated in Fig. 1.

Suppose that a given image $\mathbf{f}=\{\mathrm{f}(x, y), x=1, \ldots, M, y=$ $1, \ldots N$ has been corrupted by additive noise as

$$
\mathbf{g}=\mathbf{f}+\mathbf{n},
$$

where $\mathbf{g}$ and $\mathbf{f}$ are the noisy image and the original image, respectively, $\mathbf{n}$ represents the noise and is modeled as independent and identically distributed (i.i.d). Gaussian with zero mean and standard deviation $\sigma$, denoted as $\mathcal{N}\left(0, \sigma^{2}\right)$.

The multiscale decomposition $\mathbf{G}$ of the noisy image $\mathbf{g}$ at stage 1 can be obtained through the application of the discrete wavelet transform $\mathbf{W}$, expressed as

$$
\mathbf{G}=\mathbf{W}(\mathbf{g})
$$




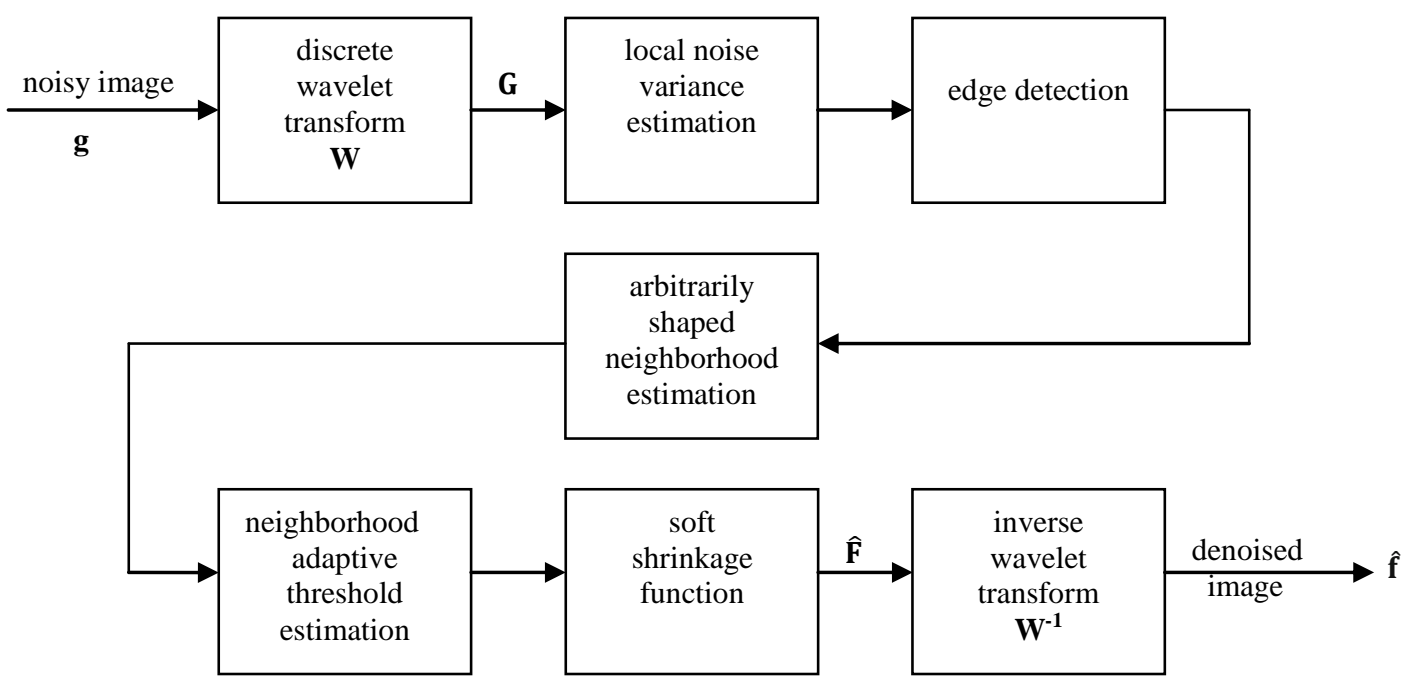

Fig 1: Proposed denoising method

The discrete wavelet transform decomposes an image into different frequency subbands labeled as $L L_{J}, L H_{j}, H L_{j}$, and $H H_{j}, j=1,2, \ldots \ldots, J$, where $j$ is the scale and $J$ is the coarsest scale. The larger $j$ is, the coarser the scale is.

Let us use the notations $\mathbf{g}^{J}, \mathbf{S}_{1}^{j}, \mathbf{S}_{2}^{j}$ and $\mathbf{S}_{3}^{j}, j=1, \ldots \ldots$, , for the subbands $\mathrm{LL}_{j}, \mathrm{LH}_{j}, \mathrm{HL}_{j}$ and $\mathrm{HH}_{j}$, respectively. Consider the detail subband $\mathbf{S}_{l}^{j}, j=1, \ldots . ., J$ and $l=1,2,3$. Note that the additive noise model for image $\mathbf{g}$ in spatial domain in Eq. (2) is also applicable for the subband $\mathbf{S}_{l}^{j}$ in wavelet domain. Thus, we have

$$
\mathbf{S}_{l}^{j}=\mathbf{w}_{l}^{j}+\mathbf{n}_{l}^{j},
$$

where the noisy subband $\mathbf{S}_{l}^{j}$ is obtained after adding the noise $\mathbf{n}_{l}^{j}$ in its noiseless counterpart $\mathbf{w}_{l}^{j}$. The objective of the proposed locally adaptive thresholding scheme is to obtain $\widehat{\mathbf{w}}_{l}^{j}$, the estimate of $\mathbf{w}_{l}^{j}$, from its noisy observation $\mathbf{S}_{l}^{j}$. Let $y_{k} \in \mathbf{S}_{l}^{j}, w_{k} \in \mathbf{w}_{l}^{j}$ and $n_{k} \in \mathbf{n}_{l}^{j}$. Eq. (4) can be rewritten as

$y_{k}=w_{k}+n_{k}, \quad k=1 \ldots \ldots$ no. of wavelet coefficients

where $y_{k}$ is noisy observation of $w_{k}$ and $n_{k}$ is noisy sample. Thus, we have to obtain $\widehat{w}_{k} \in \widehat{\mathbf{w}}_{l}^{j}$ which is an estimate of $w_{k}$ from its noisy observation $y_{k}$.

The local noise variance $\hat{\sigma}_{\text {noise }, j}^{2}$ at stage 2 can be estimated as

$$
\hat{\sigma}_{\text {noise }, j}=\frac{\text { median }\left(\left|\mathrm{y}_{i}\right|\right)}{0.6745}, \mathrm{y}_{i} \in \mathbf{S}_{3}^{j}
$$

The expression on right-hand side of Eq. (6) is a robust median estimator generally used to estimate noise variance from highest frequency subband, i.e., the subband $\mathbf{S}_{3}^{1}$ ) [6].

An edge detection algorithm at stage 3 is used to identify edges in the image. A multiscale edge detection based on Haar wavelet transform modulus maxima is used for this purpose [24], being applied separately to each subband. The multiscale edge detection produces an edge map for each subband $\mathbf{S}_{l}^{j}$, that is, a binary image where 1 represents an active edge element and 0 represents a non-edge element.

An arbitrarily shaped window at stage 4 is estimated. The objective is to determine a reasonable window size in order to estimate the signal variance for each wavelet coefficient. We have used region merging method [11] for this purpose. Assume that there is a region $R$ including $y_{k}$. Let $r_{k, 0}, \cdots, r_{k, Q-1}$ be the disjoint partitions of region $R$, that is, $r_{k, i} \cap r_{k, j}=\emptyset, i \neq j$, and $\cup_{i} r_{k, i}=R$. In addition, only a subregion $r_{k, 0}$ includes $y_{k}$.

Starting from the subregion $r_{k, 0}$ which includes the denoising point $y_{k}$, the region in which to estimate the signal variance $\hat{\sigma}_{\text {signal }, k}^{2}$ is expanded until the homogeneity of the variance is achieved. The measure of the homogeneity is defined according to the normalized difference of variances, that is

$$
h_{k, q}=\frac{\left|\sigma_{k, q}^{2}-\sigma_{k, 0}^{2}\right|}{\sigma_{k, 0}^{2}}, q=0,1, \cdots, Q-1
$$

where $\sigma_{k, q}^{2}$ is the local variance of subregion $r_{k, q}$. Since the local mean of wavelet coefficients is very small, $\sigma_{k, q}^{2}$ is approximately calculated by

$$
\sigma_{k, q}^{2}=\frac{1}{\left|r_{k, q}\right|} \sum_{y_{m} \in r_{k, q}} y_{m}^{2}
$$

where, $\left|r_{k, q}\right|$ is the size of the subregion $r_{k, q}$.

Let $b_{k, q}$ be a binary factor indicating whether the variance $\sigma_{k, q}^{2}$ is homogenous or not with $\sigma_{k, 0}^{2}$. That is,

$$
b_{k, q}=\left\{\begin{array}{l}
1, \text { if } h_{k, q}<t_{k} \\
0, \text { otherwise }
\end{array}\right.
$$

where $t_{k}$ is the homogeneity threshold defined as

$$
t_{k}=\beta 2^{(L-l)}, l=0, \cdots, L .
$$

Therefore, an arbitrarily shaped locally adaptive neighborhood region $W(k)$ around the coefficient $y_{k}$ will be formed by merging those subregions (partitions) of region $R$ whose local variances are homogeneous with the local variance of the subregion $r_{k, 0}$. In other words, the variablesized locally adaptive window $W(k)$ will contain those subregions $r_{k, q}$ whose respective binary factor $b_{k, q}$ is 1 . Various types of locally adaptive windows are demonstrated in Fig. 2. 


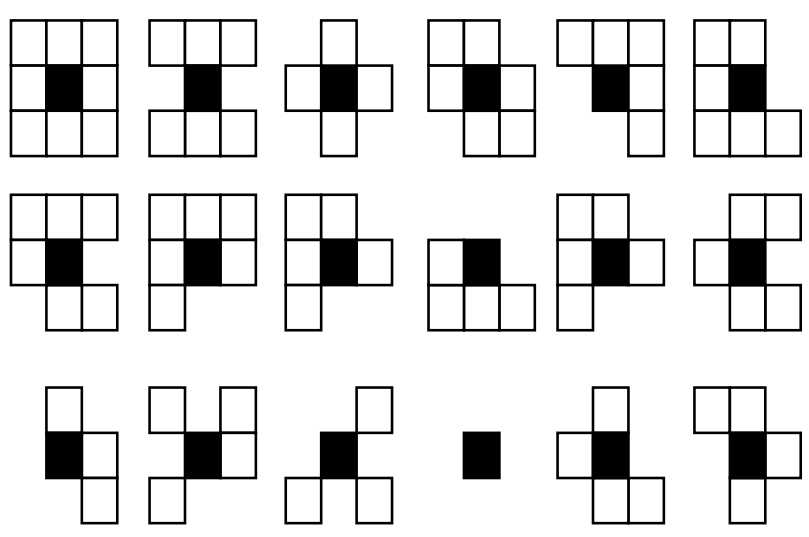

Fig. 2: Various types of locally adaptive windows. The black-colored region indicates $r_{k, 0}$.

In Eq.(10), $\beta$ is a scaling constant, $L=J-1$. Note that $l=0$ represents the finest scale and $l=L$ represents the coarsest scale of the wavelet decomposition. Then, the local variance of neighborhood window $W(k)$ is obtained as

$$
\hat{\sigma}_{W(k)}^{2}=\frac{\sum_{q=0}^{Q-1} \sigma_{k, q}^{2} \cdot b_{k, q}}{\sum_{q=0}^{Q-1} b_{k, q}}
$$

Therefore, the estimate of the signal variance $\hat{\sigma}_{\text {signal }, k}^{2}$ can be obtained as

$$
\hat{\sigma}_{\text {signal }, k}^{2}=\left(\hat{\sigma}_{W(k)}^{2}-\hat{\sigma}_{\text {noi se }, j}^{2}\right)_{+}
$$

where $(a)_{+}$is defined as

$$
(a)_{+}=\left\{\begin{array}{cc}
0, & \text { if a }<0 \\
a, & \text { otherwise }
\end{array}\right.
$$

The neighborhood adaptive threshold $\lambda_{k}$ at stage 5 to threshold the wavelet coefficient $y_{k}$ is obtained by using BayesShrink criterion [5, 17], expressed as

$$
\lambda_{k}=\frac{\widehat{\sigma}_{\text {noise }, j}^{2}}{\widehat{\sigma}_{\text {signal }, k}}
$$

Before thresholding of the coefficient $y_{k}$ by using above estimated threshold $\lambda_{k}$, we have checked its activeness toward an edge by using edge map which was computed in stage 3 . If it is related to active edge element, it must be associated with smaller threshold value. The threshold $\lambda_{k}^{\prime}$ for such coefficients proposed in our method is computed as the product between the threshold $\lambda_{k}$ and a given value $\tau$, expressed by

$$
\lambda_{k}^{\prime}=\tau \lambda_{k}
$$

that is, $\tau$ corresponds to a factor used to weight the threshold in wavelet coefficients related to edges in the image. Thus, if $y_{k}$ is related to an edge in the image, the denoised value $\widehat{w}_{k}$ is obtained by applying its corresponding threshold $\lambda_{k}^{\prime}$ according to a soft shrinkage function [16] at stage 6 as follows:

$$
\widehat{w}_{k}=T_{\text {soft }}\left(y_{k}, \lambda_{k}^{\prime}\right)=\operatorname{sgn}\left(y_{k}\right) \max \left(\left|y_{k}\right|-\lambda_{k}^{\prime}, 0\right)
$$

Otherwise, the denoised value $\widehat{w}_{k}$ is obtained by applying the threshold $\lambda_{k}$ as follows:

$$
\widehat{w}_{k}=T_{\text {soft }}\left(y_{k}, \lambda_{k}\right)=\operatorname{sgn}\left(y_{k}\right) \max \left(\left|y_{k}\right|-\lambda_{k}, 0\right)
$$

where $\operatorname{sgn}(x)$ function returns the sign of the parameter $x$.
By suppressing the noise in each coefficient $y_{k}$ in $\mathbf{S}_{l}^{j}$ through above described thresholding scheme, we get the corresponding denoised coefficient $\widehat{w}_{k}$. That is, the estimate $\widehat{\mathbf{w}}_{l}^{j}$ is obtained. The above procedure will be used to obtain the noiseless estimate for all the detail subbands at each level and the final thresholded result of complete image in wavelet domain can be obtained as

$$
\widehat{\mathbf{F}}=\left\{\mathbf{g}^{J} ; \widehat{\mathbf{w}}_{1}^{j} ; \widehat{\mathbf{w}}_{2}^{j} ; \widehat{\mathbf{w}}_{3}^{j}, j=J, J-1, \ldots, 1\right\}
$$

Lastly, the desired denoised image $\hat{\mathbf{f}}$ can be obtained by applying an inverse discrete wavelet transform $\mathbf{W}^{-1}$, at stage 7 , on thresholded wavelet domain image $\hat{\mathbf{F}}$.

$$
\hat{\mathbf{f}}=\mathbf{W}^{-1}(\hat{\mathbf{F}})
$$

The whole denoising algorithm is summarized in Table 1.

\section{Table1. Algorithm for proposed denoising method}

\section{Input: Noisy image $\mathbf{g}$}

Output: Denoised image $\hat{\mathbf{f}}$.

1: Obtain the $J$ - level wavelet decomposition of noisy

2: image $\mathbf{g}$ into the frequency subbands $\mathbf{g}^{J}, \mathbf{S}_{1}^{j}, \mathbf{S}_{2}^{j}, \mathbf{S}_{3}^{j}, \quad j=$

$3: 1,2, \ldots, J$ using (3).

4: for each resolution scale $(j=1 \ldots \ldots J)$ do

5: Calculate the local noise variance $\hat{\sigma}_{\text {noise }, j}^{2}$ using (6).

6: for each subband $\mathbf{S}_{l}^{j}=\mathbf{w}_{l}^{j}+\mathbf{n}_{l}^{j},(l=1,2,3)$ do

7: $\quad$ Compute edge map for $\mathbf{S}_{l}^{j}$

8: $\quad$ for each coefficient $y_{k}$ in $\mathbf{S}_{l}^{j}$ do

9: $\quad$ Determine the arbitrarily shaped locally adaptive

10: $\quad$ window $W(k)$ around $y_{k}$ using (7)-(10).

11: $\quad$ Compute the local variance $\hat{\sigma}_{W(k)}^{2}$ of the window

12: $\quad W(k)$ using $(11)$.

13: $\quad$ Compute the signal deviation $\hat{\sigma}_{\text {signal }, k}$ using (12).

14: if the coefficient $y_{k}$ is not related to an edge in the 15: $\quad$ image do

16: Obtain the corresponding threshold $\lambda_{k}$ using (14).

17: $\quad$ Get the denoised estimate $\widehat{w}_{k}$ using (17).

18: $\quad$ else

19: $\quad$ Obtain the corresponding threshold $\lambda_{k}^{\prime}$ using (15).

20: $\quad$ Get the denoised estimate $\widehat{w}_{k}$ using (16).

21: $\quad$ end if

22: $\quad$ end for

\section{3: end for}

\section{4: end for}

25: Get the thresholded wavelet output $\widehat{\mathbf{F}}$ using (18).

26: Obtain the denoised image $\hat{\mathbf{f}}$ by performing an inverse

27: wavelet transform to $\hat{\mathbf{F}}$ using (19). 


\section{EXPERIMENTAL RESULTS}

To validate the proposed denoising method, a number of experiments were conducted on several real test images corrupted by simulated additive white Gaussian noise at six

different power level $\sigma \in[10,15,20,25,30,35]$. The proposed algorithm has been iterated over ten different noise realizations for each standard deviation and the results are averaged over these ten runs. The test set comprises images from standard gray-scale image dataset [25], as well as wellknown images such as Lena, cameraman and peppers. A subset of gray-scale test images shown in Fig. 3 is considered in the subsequent discussions for testing the performance.

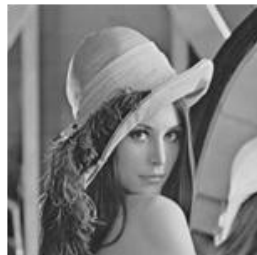

(a) Lena

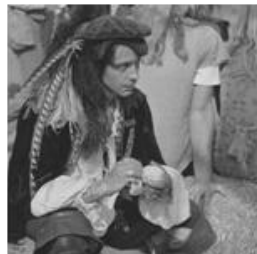

(d) Man

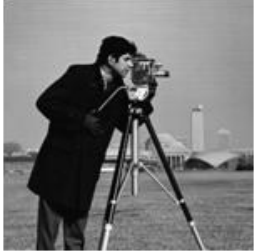

(b) Cameraman

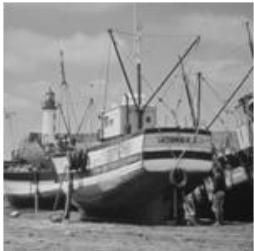

(e) Boat

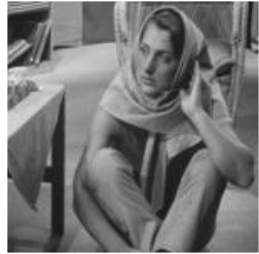

(c) Barbara

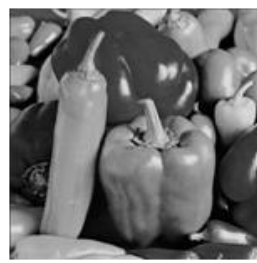

(f) Peppers
Fig. 3 Images used in experiments

\subsection{Experimental Setup}

We estimate a set of parameters used by the proposed method in section 4: wavelet transform and its number of resolution scales (decomposition levels) $J$, size of region $R$, number of subregions $Q$, scaling constant $\beta$ [Eq. (10)], $\tau$ [Eq. (15)]. These parameters are estimated using a set of images different from those shown in the comparisons. Once the parameters are set, they are kept fixed throughout the comparisons to other methods.

A set of stationary wavelets [26] from Symlet, Coeflet, Daubechies and Biothogonal families is tested for effectiveness. According to our experiments, Symlet-8 (sym8) provided better results than other wavelet bases. In addition, four resolution levels (i.e. $J=4$ ) achieved the best results. Thus, for all other wavelet-based methods (LAPB, LAWML, NASWML and Bayes), the Symlet-8 with four decomposition levels is used for fair comparison.

In our experiments, we have chosen the size of region $R$ as 9 $\times 9$ and number of subregions $Q=9$. Therefore, nine $3 \times 3$ subregions, and there exist 256 differently shaped windows. For practical purposes, all subregions were modeled in square shapes as shown in Fig. 2. The scaling constant of homogeneity threshold $t_{k}$ is set at $\beta=0.1$ (our results were insensitive to this value).

According to our experiments, the best value for $\tau$, defined in Eq. 17, is 0.8 . This shows that it is worth having a trade-off between smoothness and edge preservation. This value will be used in the remaining experiments and comparisons.

\subsection{Comparisons}

To assess the denoising effectiveness, the proposed method is compared to state-of-the-art methods, namely, LAPB [13], LAWML [10], NASWML [11] and Bayes [5]. PSNR (in dB), SSIM [27] and FOM [28] values of the denoised images relative to their original images using these methods are reported in Tables 2, 3 and 4, respectively. The best values amongst all the methods are highlighted with bold face.

The results shown in tables demonstrate that the proposed method is superior to the methods LAWML, NASWML and Bayes in regarding the PSNR and SSIM measures but sometimes these methods are competing in regarding FOM measure. When we compared with the LAPB method, the proposed method is at times contested in regarding the PSNR and SSIM measures but it almost beats LAPB method in regarding FOM measure.

In Fig. 4 which compares the PSNR performances of our method with other well-known denoising methods, starting from (a) to (f) it can be observed that the proposed method performs better in comparison to others in most of the cases. Though sometimes the LAPB method [Fig. 4 (a), (d), (e)] marginally beats the proposed one. Figures $5,6,7$ and 8 demonstrate the visual comparison among all the methods including the proposed one in respect to the sample test images: Lena, Barbara, Cameraman and Peppers, respectively.

The Bayes method tends to produce smoothened results in homogeneous regions. Nevertheless, certain features such as edges are affected. As the proposed denoising method takes into account the located edges in each high frequency subband to threshold the wavelet coefficients, it is possible to observe that such variable-sized window-based locally adaptive thresholding, effectively reduces noise while preserving features of the image. This effect can be better seen in Figs. $5(f)$ and 7 (f).

The LAPB method produces a similar result on edges. However it can be perceived from Figs. 5, 6, 7 and 8, that the proposed method outperforms LAPB in homogeneous regions, producing smoother results. That can be clearly observed in the various smooth regions in Fig. 8.

The LAWML and Bayes methods fail to smoothen up images when noise increases to higher levels. These produce good results at lower $\sigma$ values but give poor denoised images at higher noise levels. The proposed method overcomes such problem as some crucial parameters are set based on noise levels.

\section{CONCLUSIONS}

This paper presented a new edge-preserving image denoising method in wavelet domain. A new locally adaptive thresholding scheme which employs arbitrarily shaped (variable-sized) local windows to estimate the thresholding parameters, is used to effectively suppress Gaussian noise while preserving relevant features of the original image. The region merging technique is used to obtain variable-sized locally adaptive window. In our approach, an adaptive threshold is computed in neighborhood-dependent manner to effectively characterize local features of the image. This neighborhood-dependent thresholding is obtained based on the calculation of local noise variance and edge strength.

The proposed method has several desirable features. First, with the approach of using variable-sized local neighborhood window we can obtain accurate local statistics of the image. Second, estimating the term noise variance, used in the 
computation of thresholds, locally at each resolution scale makes it more beneficial as it takes the noise strength at that scale into consideration. Third, taking the edge strength of each pixel into consideration during its thresholding gives rise to better edge-preservation.

\begin{tabular}{|c|c|c|c|c|c|}
\hline \multicolumn{6}{|c|}{$\begin{array}{c}\text { Table 2. Performance of various methods as measured by PSNR } \\
\text { Methods }\end{array}$} \\
\hline & $\begin{array}{l}\text { LAPB } \\
\text { method }\end{array}$ & $\begin{array}{l}\text { LAWML } \\
\text { method }\end{array}$ & $\begin{array}{l}\text { NASWML } \\
\text { method }\end{array}$ & $\begin{array}{l}\text { Bayes } \\
\text { method }\end{array}$ & $\begin{array}{l}\text { Proposed } \\
\text { method }\end{array}$ \\
\hline \multicolumn{6}{|l|}{ Lena } \\
\hline$\sigma=10$ & 34.36 & 34.35 & 34.43 & 33.54 & 34.67 \\
\hline$\sigma=15$ & 32.56 & 32.31 & 32.57 & 31.32 & 32.78 \\
\hline$\sigma=20$ & 31.74 & 30.80 & 31.01 & 30.10 & 30.98 \\
\hline$\sigma=25$ & 30.85 & 29.76 & 29.87 & 29.43 & 30.42 \\
\hline$\sigma=30$ & 29.71 & 28.89 & 28.91 & 28.56 & 29.87 \\
\hline$\sigma=35$ & 28.73 & 28.15 & 28.16 & 28.19 & 28.95 \\
\hline \multicolumn{6}{|l|}{ Cameraman } \\
\hline$\sigma=10$ & 36.29 & 36.01 & 36.33 & 35.16 & 36.57 \\
\hline$\sigma=15$ & 33.97 & 33.65 & 34.01 & 32.93 & 34.39 \\
\hline$\sigma=20$ & 32.51 & 32.04 & 32.46 & 31.58 & 32.65 \\
\hline$\sigma=25$ & 31.23 & 30.82 & 31.32 & 30.34 & 31.79 \\
\hline$\sigma=30$ & 30.58 & 29.81 & 30.34 & 29.54 & 30.76 \\
\hline$\sigma=35$ & 30.25 & 29.01 & 29.49 & 29.03 & 30.50 \\
\hline \multicolumn{6}{|l|}{ Barbara } \\
\hline$\sigma=10$ & 32.41 & 32.47 & 32.44 & 31.10 & 32.69 \\
\hline$\sigma=15$ & 30.76 & 30.33 & 30.61 & 29.54 & 31.47 \\
\hline$\sigma=20$ & 29.16 & 28.95 & 29.24 & 27.50 & 29.48 \\
\hline$\sigma=25$ & 27.82 & 27.99 & 28.32 & 26.67 & 28.60 \\
\hline$\sigma=30$ & 27.51 & 27.25 & 27.55 & 25.47 & 27.78 \\
\hline$\sigma=35$ & 26.85 & 26.62 & 26.91 & 24.97 & 27.11 \\
\hline \multicolumn{6}{|l|}{ Man } \\
\hline$\sigma=10$ & 31.42 & 31.20 & 31.46 & 31.44 & 31.80 \\
\hline$\sigma=15$ & 29.68 & 29.64 & 29.73 & 29.76 & 30.27 \\
\hline$\sigma=20$ & 28.86 & 28.34 & 28.59 & 28.50 & 28.70 \\
\hline$\sigma=25$ & 28.10 & 27.34 & 27.74 & 27.36 & 27.89 \\
\hline$\sigma=30$ & 27.44 & 26.54 & 27.05 & 27.04 & 27.24 \\
\hline$\sigma=35$ & 26.72 & 25.92 & 26.44 & 25.94 & 26.86 \\
\hline \multicolumn{6}{|l|}{ Boat } \\
\hline$\sigma=10$ & 32.69 & 32.74 & 32.70 & 32.07 & 32.89 \\
\hline$\sigma=15$ & 31.45 & 30.65 & 30.97 & 30.14 & 31.70 \\
\hline$\sigma=20$ & 30.59 & 29.37 & 29.59 & 28.80 & 30.25 \\
\hline$\sigma=25$ & 29.34 & 28.37 & 28.56 & 27.48 & 28.80 \\
\hline$\sigma=30$ & 28.51 & 27.56 & 27.60 & 26.77 & 28.14 \\
\hline$\sigma=35$ & 27.76 & 26.88 & 27.01 & 26.25 & 27.20 \\
\hline \multicolumn{6}{|l|}{ Peppers } \\
\hline$\sigma=10$ & 32.82 & 32.53 & 32.59 & 32.38 & 32.96 \\
\hline$\sigma=15$ & 31.84 & 31.16 & 31.49 & 30.51 & 32.17 \\
\hline$\sigma=20$ & 30.75 & 30.05 & 30.38 & 29.59 & 31.28 \\
\hline$\sigma=25$ & 29.48 & 29.17 & 29.51 & 28.88 & 29.71 \\
\hline$\sigma=30$ & 29.02 & 28.42 & 28.64 & 27.95 & 29.31 \\
\hline$\sigma=35$ & 28.68 & 27.78 & 27.87 & 26.15 & 28.88 \\
\hline
\end{tabular}

Table 3. Performance of various methods as measured by SSIM

\begin{tabular}{|c|c|c|c|c|c|}
\hline & \multicolumn{5}{|c|}{ Methods } \\
\hline & $\begin{array}{l}\text { LAPB } \\
\text { method }\end{array}$ & $\begin{array}{c}\text { LAWML } \\
\text { method }\end{array}$ & $\begin{array}{l}\text { NASWML } \\
\text { method }\end{array}$ & $\begin{array}{l}\text { Bayes } \\
\text { method }\end{array}$ & $\begin{array}{c}\text { Proposed } \\
\text { method }\end{array}$ \\
\hline \multicolumn{6}{|l|}{ Lena } \\
\hline$\sigma=10$ & 0.90 & 0.89 & 0.90 & 0.87 & 0.91 \\
\hline$\sigma=15$ & 0.88 & 0.85 & 0.86 & 0.83 & 0.88 \\
\hline$\sigma=20$ & 0.84 & 0.82 & 0.82 & 0.80 & 0.84 \\
\hline$\sigma=25$ & 0.82 & 0.78 & 0.79 & 0.78 & 0.84 \\
\hline$\sigma=30$ & 0.81 & 0.74 & 0.75 & 0.76 & 0.80 \\
\hline$\sigma=35$ & 0.78 & 0.70 & 0.71 & 0.74 & 0.78 \\
\hline \multicolumn{6}{|l|}{ Cameraman } \\
\hline$\sigma=10$ & 0.94 & 0.92 & 0.93 & 0.89 & 0.94 \\
\hline$\sigma=15$ & 0.90 & 0.87 & 0.89 & 0.84 & 0.90 \\
\hline$\sigma=20$ & 0.88 & 0.83 & 0.84 & 0.80 & 0.87 \\
\hline$\sigma=25$ & 0.84 & 0.78 & 0.80 & 0.76 & 0.84 \\
\hline$\sigma=30$ & 0.82 & 0.73 & 0.75 & 0.74 & 0.81 \\
\hline$\sigma=35$ & 0.81 & 0.69 & 0.70 & 0.59 & 0.81 \\
\hline
\end{tabular}




\begin{tabular}{|c|c|c|c|c|c|}
\hline \multicolumn{6}{|l|}{ Barbara } \\
\hline$\sigma=10$ & 0.91 & 0.91 & 0.91 & 0.85 & 0.91 \\
\hline$\sigma=15$ & 0.87 & 0.87 & 0.88 & 0.79 & 0.88 \\
\hline$\sigma=20$ & 0.84 & 0.83 & 0.84 & 0.74 & 0.84 \\
\hline$\sigma=25$ & 0.80 & 0.79 & 0.80 & 0.70 & 0.80 \\
\hline$\sigma=30$ & 0.77 & 0.75 & 0.76 & 0.67 & 0.77 \\
\hline$\sigma=35$ & 0.73 & 0.71 & 0.71 & 0.64 & 0.74 \\
\hline \multicolumn{6}{|l|}{ Man } \\
\hline$\sigma=10$ & 0.88 & 0.86 & 0.86 & 0.86 & 0.87 \\
\hline$\sigma=15$ & 0.84 & 0.81 & 0.82 & 0.80 & 0.83 \\
\hline$\sigma=20$ & 0.79 & 0.76 & 0.77 & 0.75 & 0.80 \\
\hline$\sigma=25$ & 0.76 & 0.72 & 0.73 & 0.71 & 0.76 \\
\hline$\sigma=30$ & 0.73 & 0.68 & 0.69 & 0.69 & 0.71 \\
\hline$\sigma=35$ & 0.71 & 0.64 & 0.65 & 0.67 & 0.69 \\
\hline \multicolumn{6}{|l|}{ Boat } \\
\hline$\sigma=10$ & 0.91 & 0.90 & 0.90 & 0.86 & 0.91 \\
\hline$\sigma=15$ & 0.88 & 0.85 & 0.86 & 0.80 & 0.87 \\
\hline$\sigma=20$ & 0.84 & 0.80 & 0.81 & 0.76 & 0.84 \\
\hline$\sigma=25$ & 0.81 & 0.76 & 0.77 & 0.73 & 0.80 \\
\hline$\sigma=30$ & 0.77 & 0.71 & 0.73 & 0.71 & 0.77 \\
\hline$\sigma=35$ & 0.74 & 0.67 & 0.69 & 0.68 & 0.74 \\
\hline \multicolumn{6}{|l|}{ Peppers } \\
\hline$\sigma=10$ & 0.88 & 0.85 & 0.86 & 0.84 & 0.86 \\
\hline$\sigma=15$ & 0.84 & 0.81 & 0.82 & 0.79 & 0.84 \\
\hline$\sigma=20$ & 0.81 & 0.77 & 0.79 & 0.76 & 0.82 \\
\hline$\sigma=25$ & 0.78 & 0.73 & 0.75 & 0.73 & 0.80 \\
\hline$\sigma=30$ & 0.76 & 0.69 & 0.71 & 0.71 & 0.76 \\
\hline$\sigma=35$ & 0.75 & 0.66 & 0.67 & 0.56 & 0.74 \\
\hline \multicolumn{6}{|c|}{ Table 4. Performance of various methods as measured by FOM } \\
\hline & \multicolumn{5}{|c|}{ Methods } \\
\hline & LAPB & LAWML & NASWML & Bayes & Proposed \\
\hline & method & method & method & method & method \\
\hline \multicolumn{6}{|l|}{ Lena } \\
\hline$\sigma=10$ & 0.89 & 0.88 & 0.86 & 0.89 & 0.89 \\
\hline$\sigma=15$ & 0.86 & 0.87 & 0.85 & 0.81 & 0.86 \\
\hline$\sigma=20$ & 0.85 & 0.83 & 0.84 & 0.84 & 0.84 \\
\hline$\sigma=25$ & 0.82 & 0.79 & 0.82 & 0.76 & 0.82 \\
\hline$\sigma=30$ & 0.78 & 0.75 & 0.76 & 0.66 & 0.79 \\
\hline$\sigma=35$ & 0.75 & 0.76 & 0.72 & 0.67 & 0.76 \\
\hline \multicolumn{6}{|l|}{ Cameraman } \\
\hline$\sigma=10$ & 0.90 & 0.92 & 0.89 & 0.91 & 0.90 \\
\hline$\sigma=15$ & 0.87 & 0.85 & 0.88 & 0.87 & 0.85 \\
\hline$\sigma=20$ & 0.82 & 0.84 & 0.82 & 0.74 & 0.84 \\
\hline$\sigma=25$ & 0.78 & 0.80 & 0.76 & 0.62 & 0.81 \\
\hline$\sigma=30$ & 0.74 & 0.75 & 0.77 & 0.59 & 0.77 \\
\hline$\sigma=35$ & 0.71 & 0.71 & 0.70 & 0.57 & 0.74 \\
\hline \multicolumn{6}{|l|}{ Barbara } \\
\hline$\sigma=10$ & 0.89 & 0.90 & 0.88 & 0.91 & 0.91 \\
\hline$\sigma=15$ & 0.87 & 0.83 & 0.86 & 0.87 & 0.88 \\
\hline$\sigma=20$ & 0.81 & 0.83 & 0.85 & 0.83 & 0.85 \\
\hline$\sigma=25$ & 0.81 & 0.82 & 0.84 & 0.78 & 0.84 \\
\hline$\sigma=30$ & 0.79 & 0.81 & 0.79 & 0.72 & 0.81 \\
\hline
\end{tabular}



$\sigma=35$
0.78
0.79

0.81

0.69

0.80

Man

$\begin{array}{ll}\sigma=10 & 0.86 \\ \sigma=15 & 0.82 \\ \sigma=20 & 0.80 \\ \sigma=25 & 0.78 \\ \sigma=30 & \mathbf{0 . 7 6} \\ \sigma=35 & 0.73\end{array}$

0.87

0.84

0.91

0.90

82

0.84

0.81

0.88

0.85

0.78

0.84

0.83

0.74

0.77

0.80

0.79

0.76

0.76

0.72

0.76

0.74

Boat

$\begin{array}{ll}\sigma=10 & 0.91 \\ \sigma=15 & 0.87 \\ \sigma=20 & 0.86 \\ \sigma=25 & \mathbf{0 . 8 6} \\ \sigma=30 & 0.81 \\ \sigma=35 & \mathbf{0 . 8 1}\end{array}$

0.91

0.90

0.92

0.92

0.90

0.87

0.90

0.85

0.89

$0.86-0.83$

0.86

0.86

0.84

0.83

0.83

0.84

0.81

0.82

0.78

0.81

Peppers

$\begin{array}{lll}\sigma=10 & \mathbf{0 . 8 8} & 0.86 \\ \sigma=15 & 0.83 & \mathbf{0 . 8 5} \\ \sigma=20 & 0.82 & 0.81 \\ \sigma=25 & 0.78 & 0.74 \\ \sigma=30 & 0.76 & 0.76 \\ \sigma=35 & 0.72 & 0.71\end{array}$

0.86

0.83

0.85

0.86

0.82

0.81

0.85

$\begin{array}{ll}0.81 & \mathbf{0 . 8 3}\end{array}$

0.80

0.83

0.80

0.75

0.80

0.74

0.65

0.77

0.67

0.52

0.73

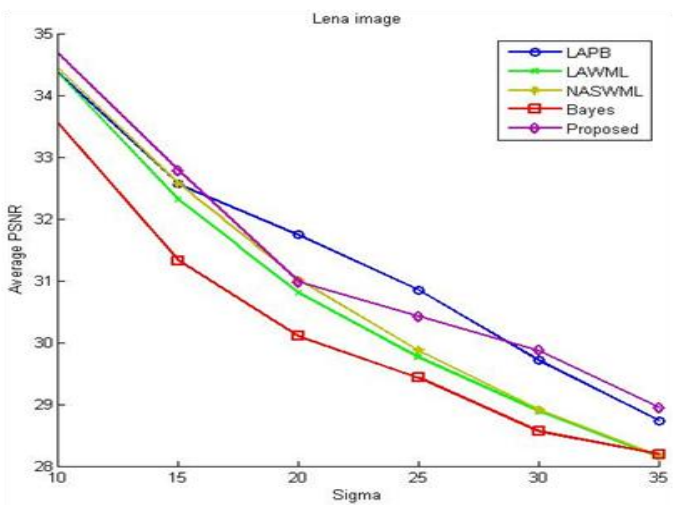

a

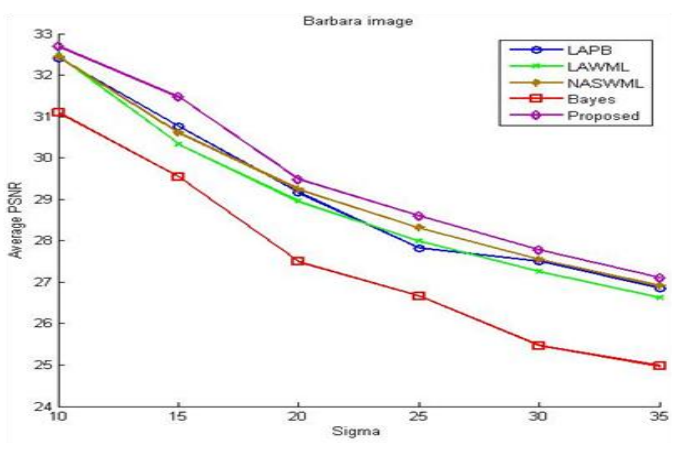

b

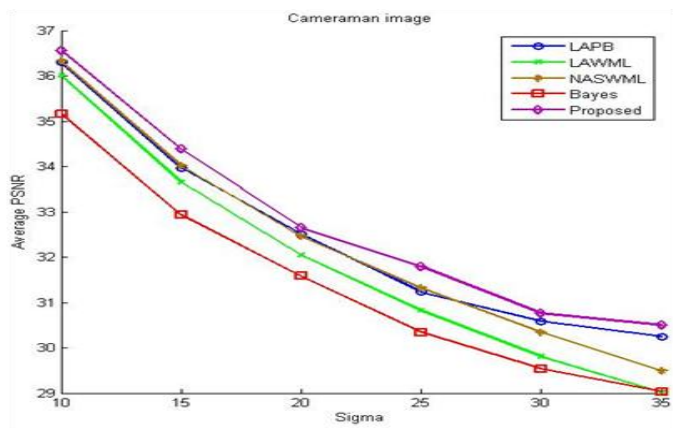

c

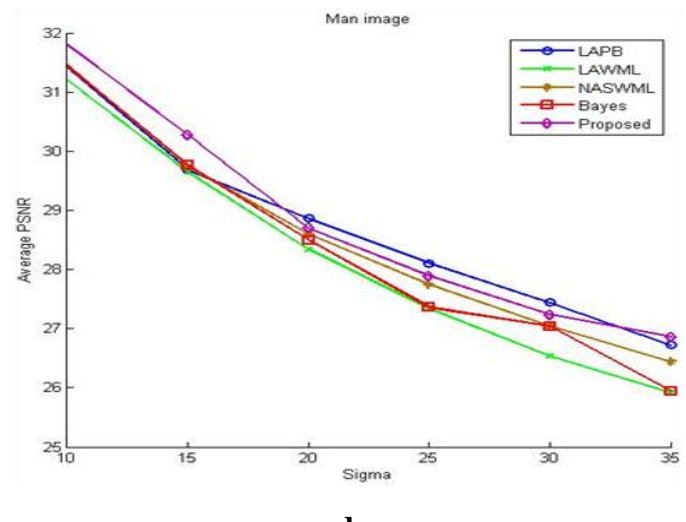

d 


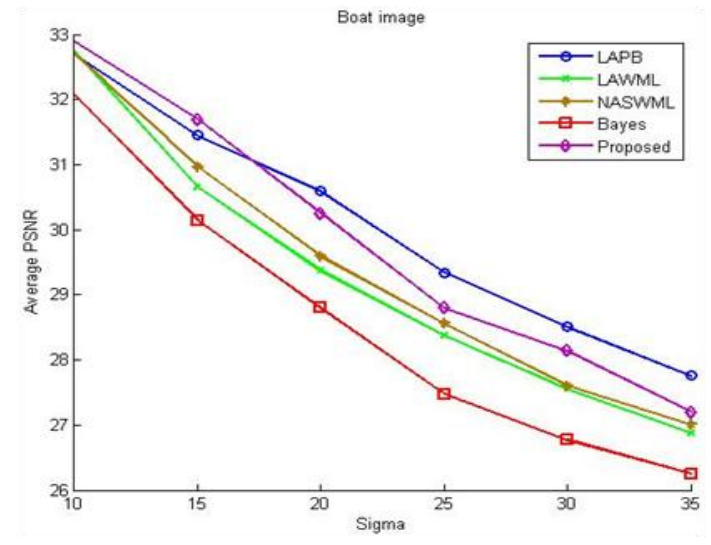

e

Fig. 4 PSNR performance graphs for test images

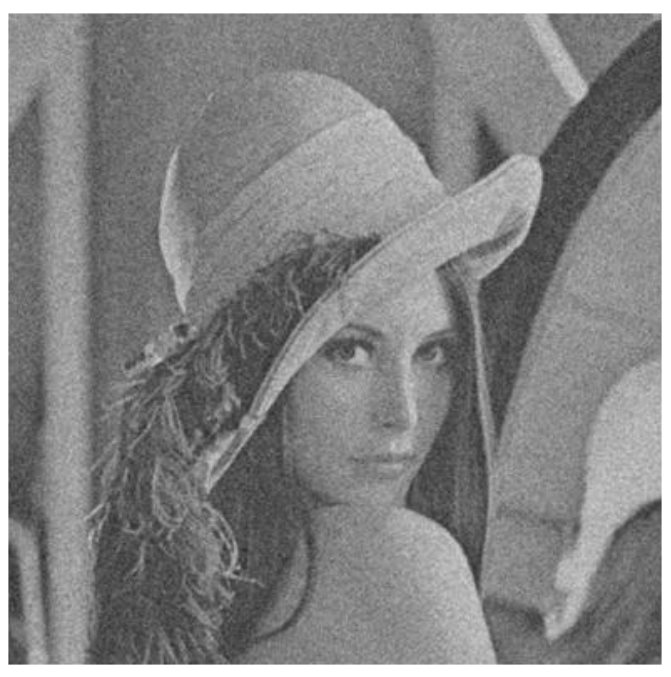

(a) Noisy $(\sigma=30)$

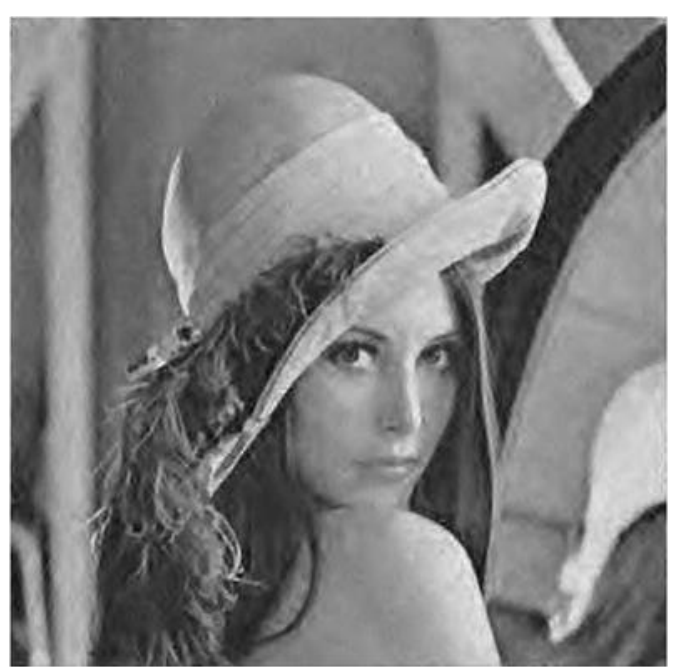

(b) LAPB

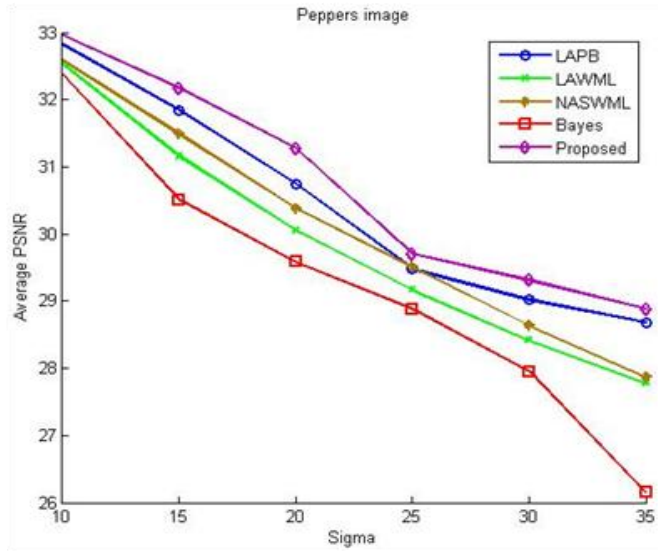

f

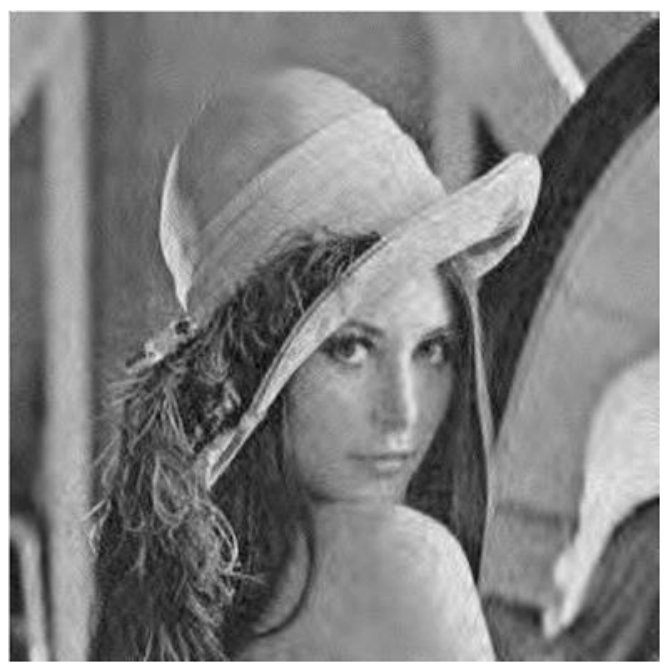

(c) LAWML

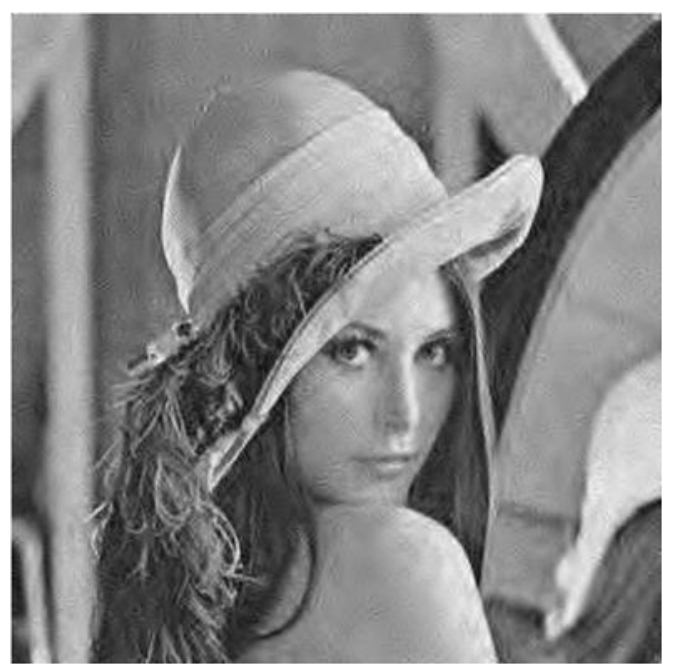

(d) NASWML 


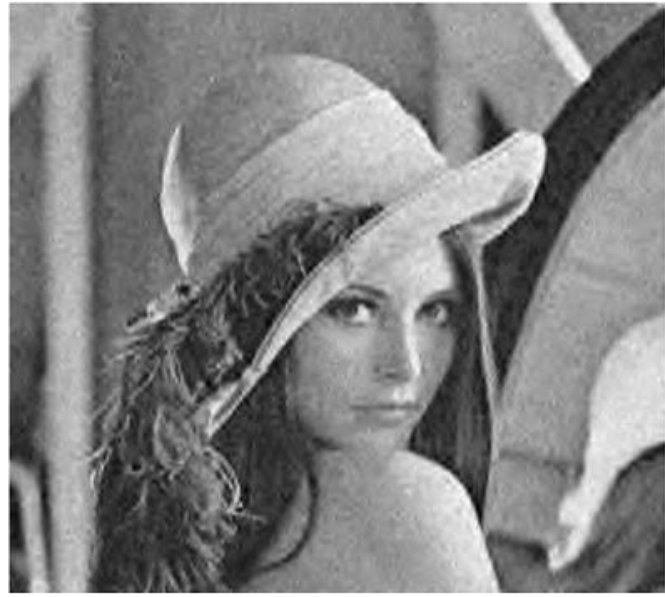

(e) Bayes

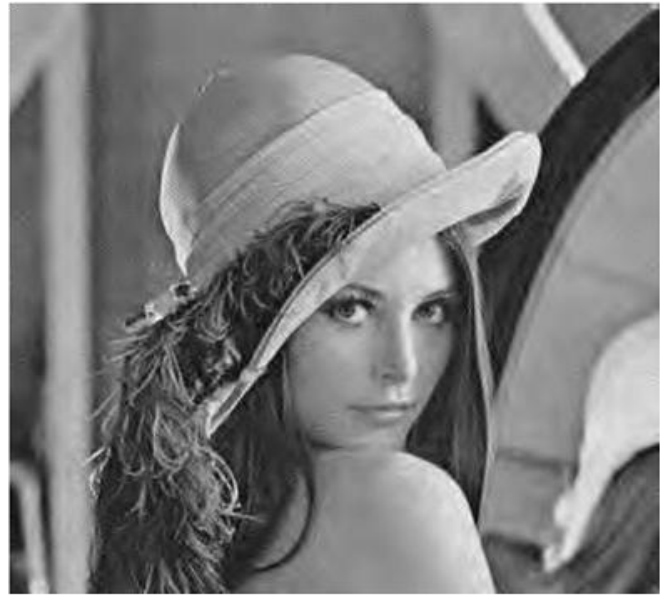

(f) Proposed

Fig. 5 Denoising results for Lena image

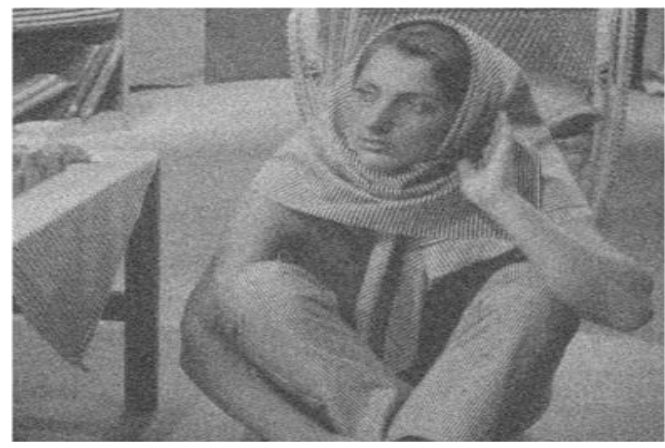

(a) Noisy $(\sigma=30)$

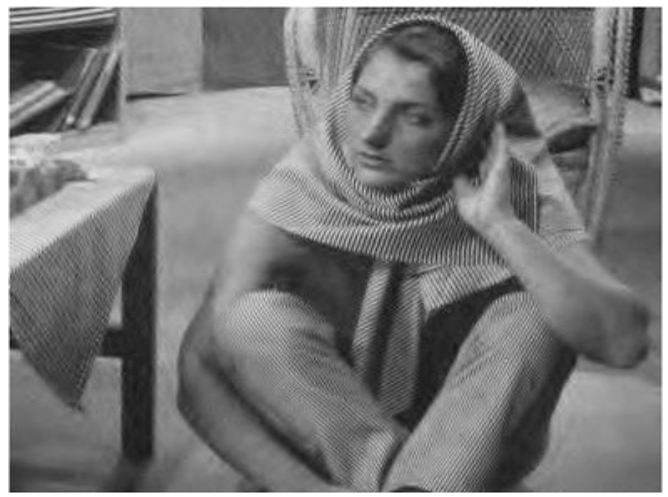

(b) LAPB

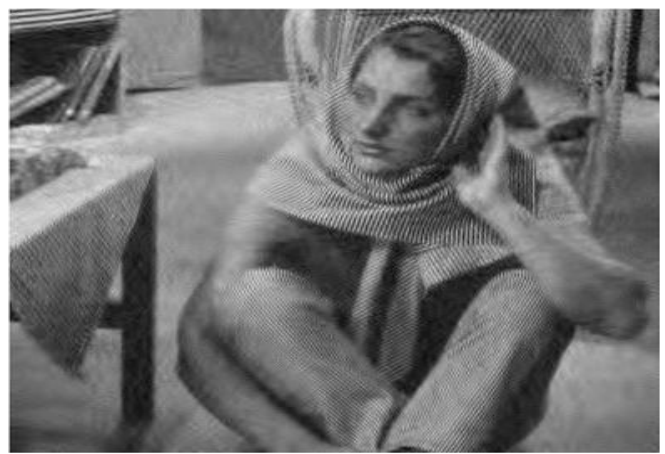

(c) LAWML

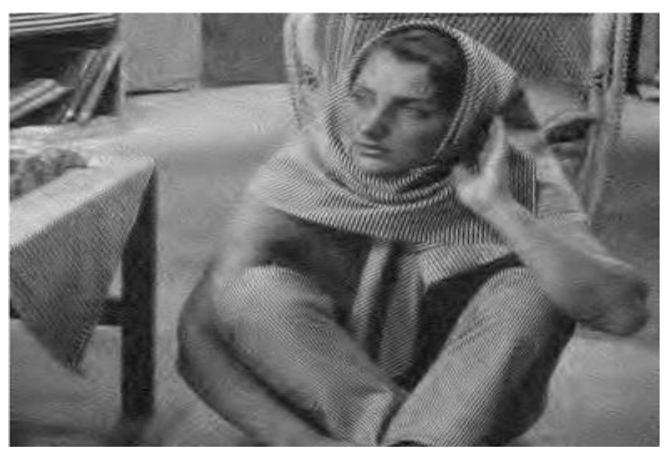

(d) NASWML

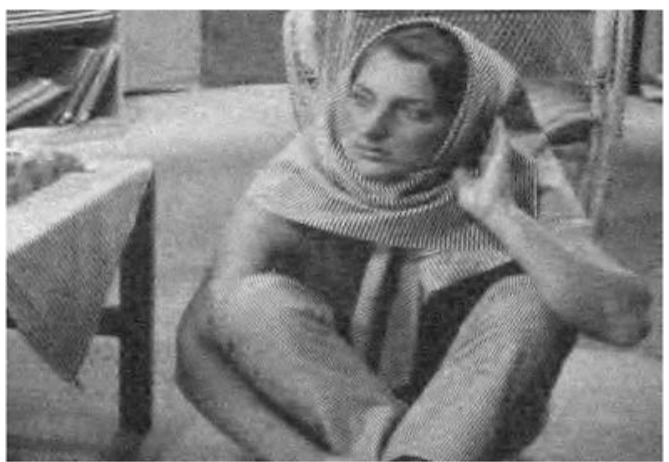

(e) Bayes

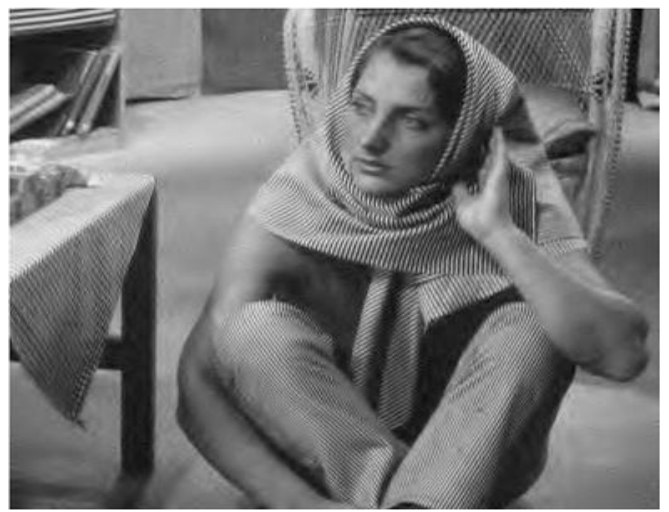

(f) Proposed

Fig. 6 Denoising results for Barbara image 


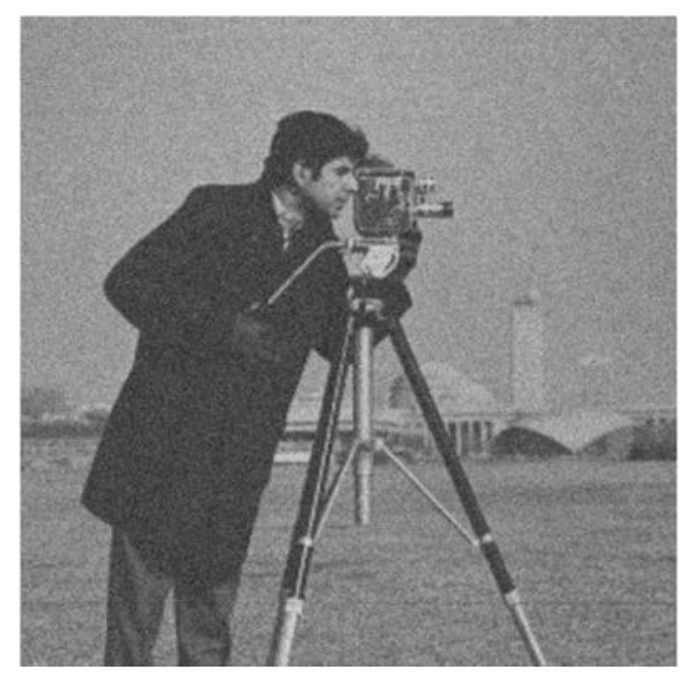

(a) Noisy $(\sigma=30)$

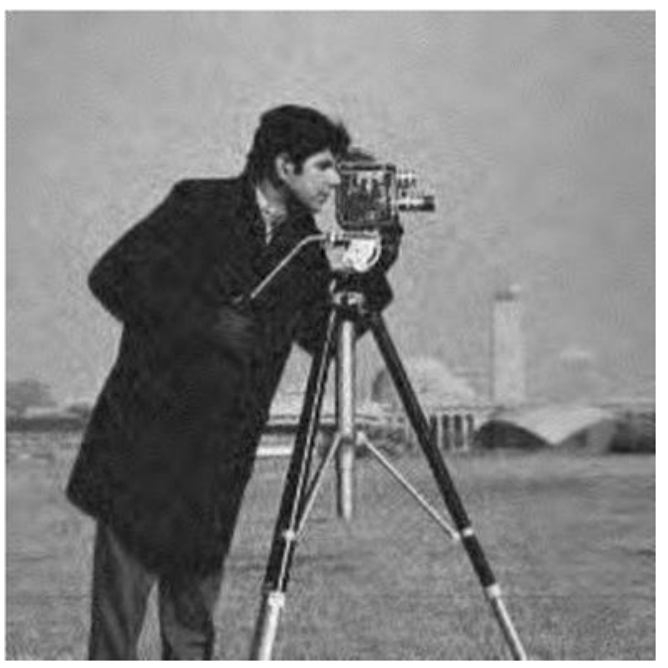

(c) LAWML

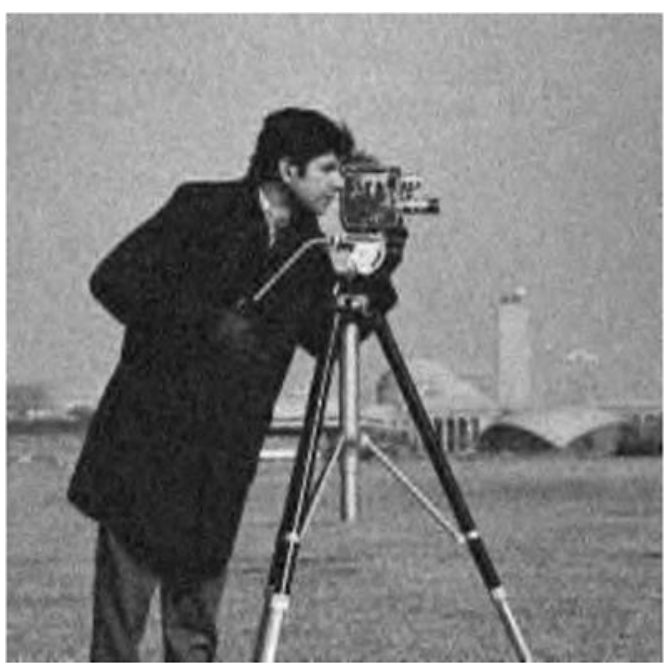

(e) Bayes

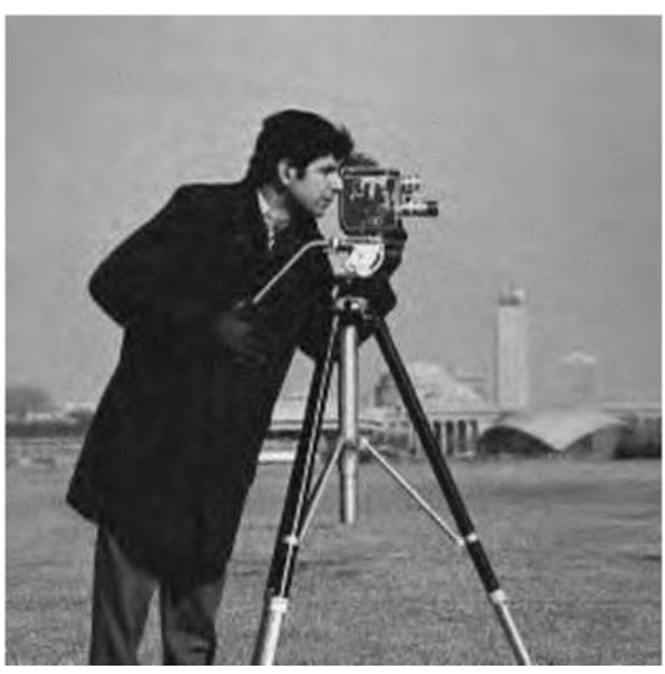

(b) LAPB

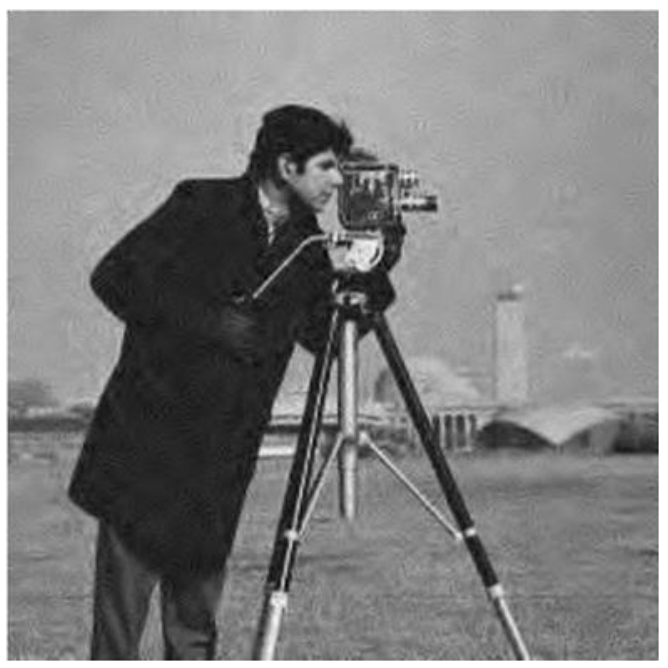

(d) NASWML

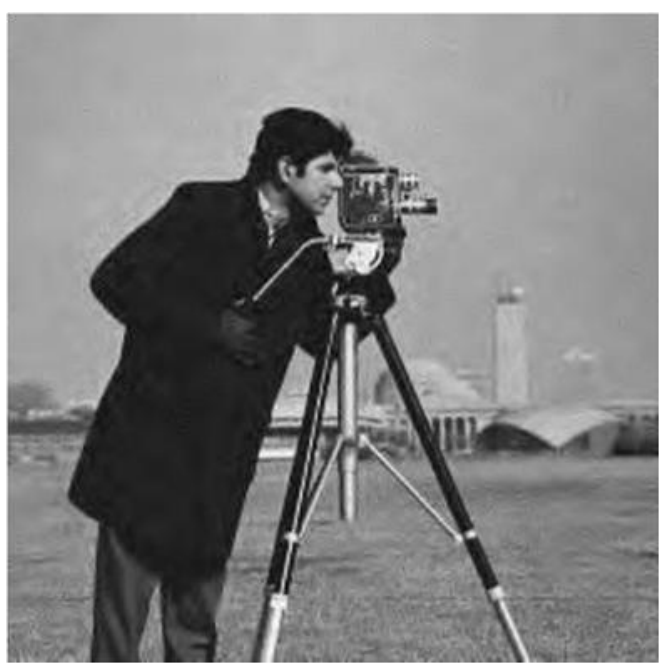

(f) Proposed

Fig. 7 Denoising results for Cameraman image 


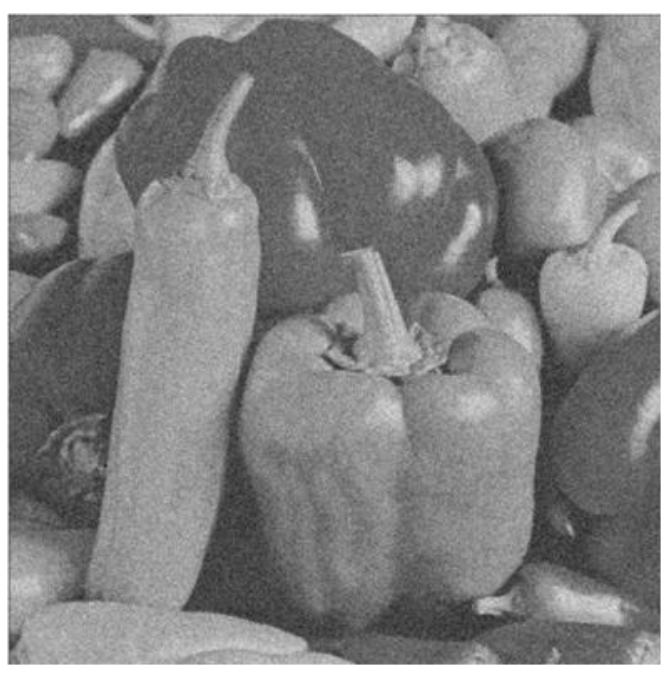

(a) Noisy $(\sigma=30)$

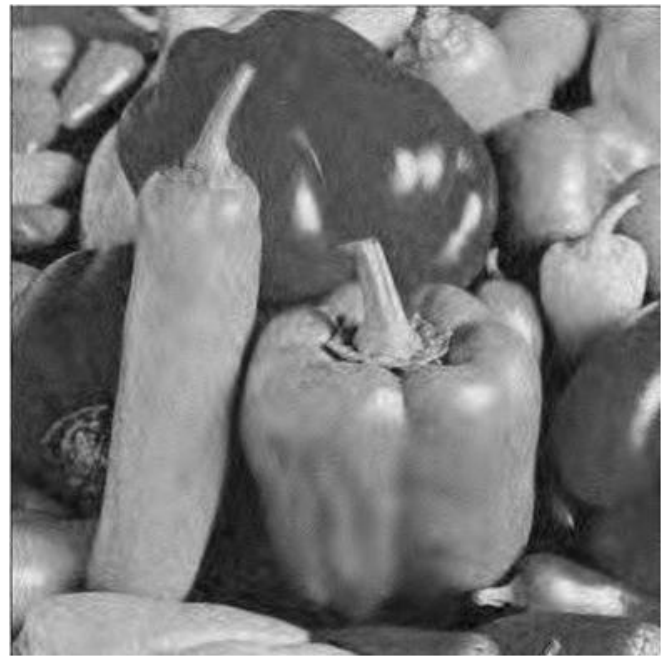

(c) LAWML

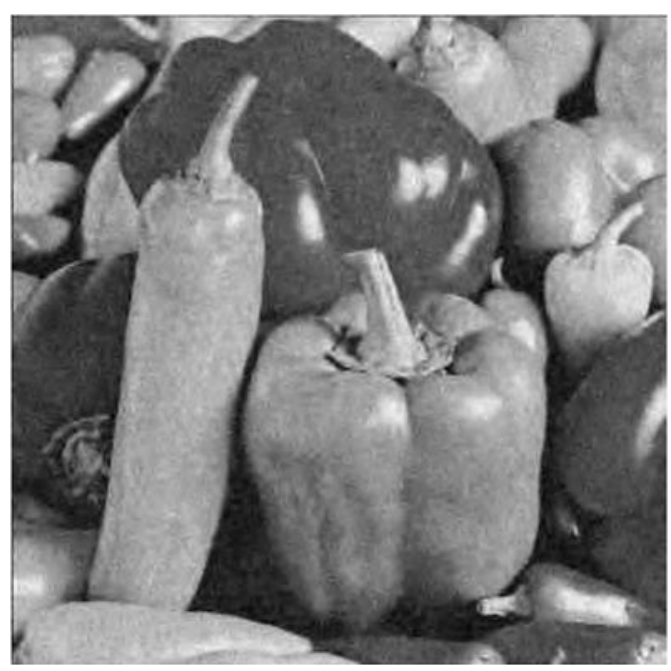

(e) Bayes

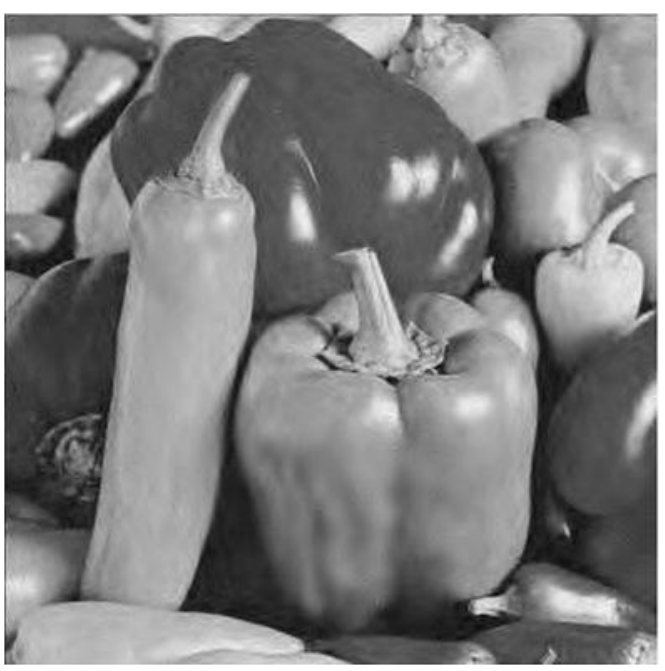

(b) LAPB

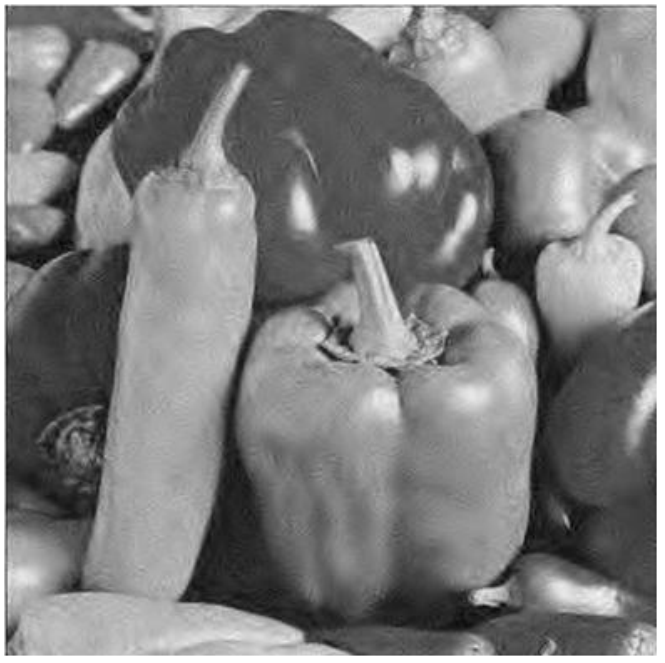

(d) NASWML

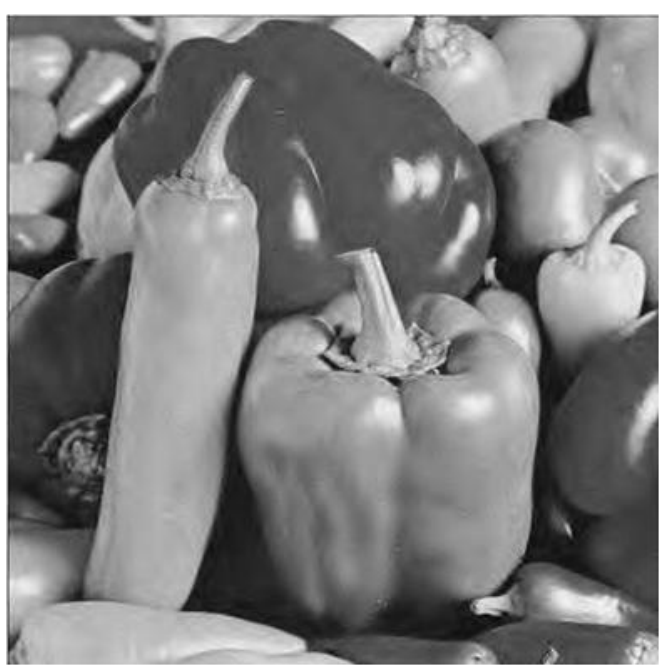

(f) Proposed

Fig. 8 Denoising results for Peppers image 


\section{REFERENCES}

[1] Gonzalez, R. C. and Woods, R. E. (2008), "Digital image processing", $3^{\text {rd }}$ edn., Prentice-Hall, Upper Saddle River.

[2] Shapiro, L. and Stockman, G. (2001). Computer Vision. Prentice-Hall.

[3] Jain, P. and Tyagi, V. (2013), "Spatial and frequency domain filters for restoration of noisy images", IETE Journal of Education, 54(2), 108-116.

[4] Jain, P. and Tyagi, V. (2014), "A Survey of EdgePreserving Image Denoising Methods", Information System Frontiers. DOI: 10.1007/s10796-014-9527-0.

[5] Chang, S., Yu, B. and Vetterli, M. (2000), "Adaptive wavelet thresholding for image denoising and compression", IEEE Transaction on Image Processing, 9(9), 1532-1546.

[6] Donoho, D. L. and Johnstone, I. M. (1994), "Ideal spatial adaptation via wavelet shrinkage", Biometrika 81, 425455.

[7] Donoho, D. L. and Johnstone, I. M. (1995), "Adapting to unknown smoothness via wavelet shrinkage", Journal of the American Statistical Association, 90(432), 12001224.

[8] Sendur, L. and Selesnick, I. W. (2002), "Bivariate shrinkage with local variance estimation", IEEE Signal Processing Letter, 9(12), 438-441.

[9] Portilla, J., Strela, V., Wainwright, M. and Simoncelli, E. (2003), "Image denoising using scale mixtures of Gaussians in the wavelet domain", IEEE Transaction of Image Processing, 12(11), 1338-1351.

[10] Mihcak, M. K., Kozintsev, I., Ramchandran, K. and Moulin, P. (1999), "Low complexity image denoising based on statistical modeling of wavelet coefficients", IEEE Signal Processing Letter, 6(12), 300-303.

[11] Eom, I. K. and Kim, Y. S. (2004), "Wavelet-based denoising with nearly arbitrary shaped windows", IEEE Signal Processing Letter, 11(2), 937-940.

[12] Jain, P. and Tyagi, V. (2014), "An adaptive edgepreserving image denoising technique using tetrolet transforms", The Visual Computer. DOI: 10.1007/s00371-014-0993-7.

[13] Jain, P. and Tyagi, V. (2015), "LAPB: Locally adaptive patch-based wavelet domain edge-preserving image denoising", Information Sciences, 294, 164-181. DOI: 10.1016/j.ins.2014.09.060.

[14] Silva, R. D., Minetto, R., Schwartz, W. R. and Pedrini, H. (2012), "Adaptive edge-preserving image denoising using wavelet transforms", Pattern Analysis and Application. DOI: 10.1007/s10044-012-0266-x.

[15] Chang, S., Yu, B. and Vetterli, M. (2000), "Spatially adaptive wavelet thresholding based on context modeling for image denoising", IEEE Transactions on Image Processing, 9(9), 1522-1531.

[16] Donoho, D. L. (1995), "De-noising by softthresholding", IEEE Transactions on Information Theory, 41(3), 613-627.

[17] Chipman, H., Kolaczyk, E. and McCulloc, R. (1997), "Adaptive Bayesian wavelet shrinkage", Journal of the American Statistical Association, 440(92), 1413-1421.

[18] Nason, G. P. (1996), "Wavelet shrinkage by crossvalidation", Journal of the Royal Statistical Society, B $58,463-479$.

[19] Weyrich, N. and Warhola, G. T. (1998), "Wavelet shrinkage and generalized cross validation for image denoising", IEEE Transactions on Image Processing, $7(1), 82-90$.

[20] Mihcak, M. K., Kozintsev, I. and Ramchandran, K. (1999), "Spatially adaptive statistical modeling of wavelet image coefficients and its application to denoising", In Proceeding of IEEE International Conference of Acoustics, Speech and Signal Processing, 6, 3253-3256.

[21] Park, J. M., Song, W. J. and Pearlman, W. A. (1999), "Speckle filtering of SAR images based on adaptive windowing", In Proceeding IEE Vision, Image and Signal Processing, 146(33), 191-197.

[22] Boykov, Y., Veksler, P. and Zabih, R. (1998), "A variable window approach to early vision", IEEE Transaction on Pattern Analysis and Machine Intelligence, 20, 1283-1294.

[23] Balan, P. and Mather, P. M. (2001), "An adaptive filter for removal of noise in interferometrically derived digital elevation models", In Proceeding IEEE International Symposium Geoscience and Remote Sensing, 6, 2529_ 2531.

[24] Mallat, S. (1989), "A theory for multiresolution signal decomposition: the wavelet representation", IEEE Transaction on Pattern Analysis and Machine Intelligence, 11(7), 674-693.

[25] http://decsai.ugr.es/cvg/CG/base.htm

[26] Nason, G. P. and Silverman, B. W. (1995), "The stationary wavelet transform and some statistical applications", In Lecture notes in statistics: wavelets and statistics, Springer-Verlag, Berlin, 281-300.

[27] Wang, Z., Bovik, A., Sheikh, H. and Simoncelli, E. (2004), "Image quality assessment: from error visibility to structural similarity", IEEE Transactions on Image Processing, 13(4), 600-612.

[28] Pratt, W. (2007), "Digital image processing", 4th edn., Wiley, New York. 